\title{
A New Family of Weak Estimators for Training in Non-stationary Distributions
}

\author{
B. John Oommen ${ }^{1, \star}$ and Luis Rueda ${ }^{2, \star \star}$ \\ ${ }^{1}$ School of Computer Science, Carleton University \\ 1125 Colonel By Dr., Ottawa, ON, K1S 5B6, Canada \\ oommen@scs.carleton.ca \\ 2 School of Computer Science, University of Windsor \\ 401 Sunset Avenue, Windsor, ON, N9B 3P4, Canada \\ Irueda@uwindsor.ca
}

\begin{abstract}
In this paper, we formally present a novel estimation method, referred to as the Stochastic Learning Weak Estimator (SLWE), which is used to estimate the parameters of a binomial distribution, where the convergence of the estimate is weak, i.e. in law. The estimation is based on the principles of stochastic learning. Even though our new method includes a learning coefficient, $\lambda$, it turns out that the mean of the final estimate is independent of $\lambda$, the variance of the final distribution decreases with $\lambda$, and the speed decreases with $\lambda$. Similar results are true for the multinomial case. An empirical analysis on synthetic data shows the advantages of the scheme for non-stationary distributions. Conclusive results demonstrate the advantage of SLWE for a pattern-recognition problem which has direct implications in data compression. In this case, the underlying distribution in the data file to be compressed is non-stationary, and it is estimated and learnt using the principles highlighted here. By classifying its variation and using it in the compression, the superiority of the scheme is documented.
\end{abstract}

\section{Introduction}

The theory of estimation has been studied for hundreds of years $[1,15]$. It is also easy to see that the learning (training) phase of a statistical pattern recognition system is, indeed, based on estimation theory $[2,17]$. Estimation methods generally fall into various categories, including the Maximum Likelihood Estimates (MLE) and the Bayesian family of estimates.

Although the MLEs and Bayesian estimates have good computational and statistical properties, the fundamental premise for establishing the quality of estimates is based on the assumption that the parameter being estimated does not change with time. In other words, the distribution is assumed to be stationary. Thus, it is generally assumed that there is an underlying parameter $\theta$, which does not change with time, and as the number

\footnotetext{
${ }^{\star}$ Fellow of the IEEE. Partially supported by NSERC, the Natural Science and Engineering Research Council of Canada.

${ }^{\star \star}$ Member, IEEE. Partially supported by NSERC, the Natural Science and Engineering Research Council of Canada.
} 
of samples increases, we would like the estimate $\hat{\theta}$ to converge to $\theta$ with probability one, or in a mean square sense.

There are numerous problems which we have recently encountered, where these strong estimators pose a real-life concern. One scenario occurs in pattern classification involving moving scenes. We also encounter the same situation when we want to perform adaptive compression of files which are interspersed with text, formulae, images and tables. Similarly, if we are dealing with adaptive data structures, the structure changes with the estimate of the underlying data distribution, and if the estimator used is "strong" (i.e., w. p. 1), it is hard for the learned data structure to emerge from a structure that it has converged to. Indeed, we can conclusively demonstrate that it is sub-optimal to work with strong estimators in such application domains, i.e., when the data is truly non-stationary.

In this paper, we shall present one such "weak" estimator, referred to as the Stochastic Learning Weak Estimator (SLWE), and which is developed by using the principles of stochastic learning. In essence, the estimate is updated at each instant based on the value of the current sample. However, this updating is not achieved using an additive updating rule, but rather by a multiplicative rule, akin to the family of linear actionprobability updating schemes $[6,7]$. The formal results that we have obtained for the binomial distribution are quite fascinating. Similar results have been achieved for the multinomial case, except that the variance (covariance) has not been currently derived.

The entire field of obtaining weak estimators is novel. In that sense, we believe that this paper is of a pioneering sort - we are not aware of any estimators which are computed as explained here. We also hope that this is the start of a whole new body of research, namely those involving weak estimators for various distributions, and their applications in various domains. In this regard, we are currently in the process of deriving weak estimators for various distributions. To demonstrate the power of the SLWE in real-life problems, we conclude the paper with conclusive results applicable to data compression problems, in which the underlying distribution is non-stationary.

The more detailed application of these results in adaptive data compression, statistical pattern recognition, adaptive data structures, and scene analysis is currently underway. Finally, the application of non-linear updating schemes to obtain weak estimators is also open, although some initial results are currently available.

To devise a new estimation method, we have utilized the principles of learning as achieved by the families of Variable Structure Stochastic Automata (VSSA) [3, 4, 6]. In particular, the learning we have achieved is obtained as a consequence of invoking algorithms related to families of linear schemes, such as the Linear Reward-Inaction $\left(L_{R I}\right)$ scheme. The analysis is also akin to the analysis used for these learning automata. This involves first determining the updating equations, and taking the conditional expectation of the quantity analyzed. The condition disappears when the expectation operator is invoked a second time, leading to a difference equation for the specified quantity, which equation is later explicitly solved. We have opted to use these families of VSSA in the design of our SLWE, because it turns out that the analysis is considerably simplified and (in our opinion) fairly elegant.

The question of deriving weak estimators based on the concepts of discretized LA $[8,10]$ and estimator-based LA $[5,9,11,13,14,16]$ remains open. We believe, however, 
that even if such estimator-based SLWE are designed, the analysis of their properties will not be trivial.

\section{Weak Estimators of Binomial Distributions}

Through out this paper we assume that we are estimating the parameters of a binomial/multinomial distribution. The binomial distribution is characterized by two parameters, namely, the number of Bernoulli trials, and the parameter characterizing each Bernoulli trial. In this regard, we assume that the number of observations is the number of trials. Thus, all we have to do is to estimate the Bernoulli parameter for each trial. This is what we endeavor to do using stochastic learning methods.

Let $X$ be a binomially distributed random variable, which takes on the value of either ' 1 ' or ' 2 ' . We assume that $X$ obeys the distribution $S$, where $S=\left[s_{1}, s_{2}\right]^{T}$. In other words,

$X=$ ' 1 ' with probability $s_{1}$

$=$ ' 2 ' with probability $s_{2}$,

where, $s_{1}+s_{2}=1$.

Let $x(n)$ be a concrete realization of $X$ at time ' $n$ '. The intention of the exercise is to estimate $S$, i.e., $s_{i}$ for $i=1,2$. We achieve this by maintaining a running estimate $P(n)=\left[p_{1}(n), p_{2}(n)\right]^{T}$ of $S$, where $p_{i}(n)$ is the estimate of $s_{i}$ at time ' $n$ ', for $i=1,2$. Then, the value of $p_{i}(n)$ is updated as per the following simple rule:

$$
\begin{aligned}
p_{1}(n+1) & \leftarrow \lambda p_{1}(n) & & \text { if } x(n)=2 \\
& \leftarrow 1-\lambda p_{2}(n) & & \text { if } x(n)=1 .
\end{aligned}
$$

where $\lambda$ is a user-defined parameter, $0<\lambda<1$.

In order to simplify the notation, the vector $P(n)=\left[p_{1}(n), p_{2}(n)\right]^{T}$ refers to the estimates of the probabilities of ' 1 ' and ' 2 ' occurring at time ' $n$ ', namely $p_{1}(n)$ and $p_{2}(n)$ respectively. In the interest of simplicity, we omit the index $n$, whenever there is no confusion, and thus, $P$ implies $P(n)$.

The first theorem, whose proof can be found in [12], concerns the distribution of the vector $P$ which estimates $S$ as per Equations (1) and (2). We shall state that $P$ converges in distribution. The mean of $P$ is shown to converge exactly to the mean of $S$. The proof, which is a little involved, follows the types of proofs used in the area of stochastic learning [12].

Theorem 1. Let $X$ be a binomially distributed random variable, and $P(n)$ be the estimate of $S$ at time ' $n$ '. Then, $E[P(\infty)]=S$.

The next results that we shall prove indicates that the distribution of $\mathrm{E}[P(n+1)]$ follows $\mathrm{E}[P(n)]$ in a Markovian manner. We derive the explicit Markovian dependence, and allude to the resultant properties by virtue of the ergodic nature of the Markov matrix. This leads us to two results, namely that of the limiting distribution of the chain,

\footnotetext{
${ }^{1}$ We depart from the traditional notation of the random variable taking values of ' 0 ' and ' 1 ', so that the notation is consistent when we consider the multinomial case.
} 
and that which concerns the rate of convergence of the chain. It turns out that while the former is independent of the learning parameter, $\lambda$, the latter is determined only by $\lambda$.

The reader will observe that the results we have derived are asymptotic results. In other words, the mean of $P(n)$ is shown to converge exactly to the mean of $S$. The implications of the "asymptotic" nature of the results will be clarified presently. The proofs of Theorems 2, 3 and 4 can be found in [12].

Theorem 2. Let $X$ be a binomially distributed random variable governed by the distribution $S$. If $P(n+1)$ is the estimate of $S$ at time ' $n+1$ ', $E[P(n)]$ obeys an ergodic Markov chain whose steady state distribution converges to $S$.

Theorem 3. Let $X$ be a binomially distributed random variable governed by the distribution $S$, and $P(n)$ be the estimate of $S$ at time ' $n$ ' obtained by (1) and (2). Then, the rate of convergence of $P$ to $S$ is fully determined by $\lambda$.

We now derive the explicit expression for the asymptotic variance of the SLWE. A small value of $\lambda$ leads to fast convergence and a large variance and vice versa.

Theorem 4. Let $X$ be a binomially distributed random variable governed by the distribution $S$, and $P(n)$ be the estimate of $S$ at time ' $n$ ' obtained by (1) and (2). Then, the algebraic expression for the variance of $P(\infty)$ is fully determined by $\lambda$.

When $\lambda \rightarrow 1$, it implies that the variance tends to zero, implying mean square convergence. The plot of the variance of $p_{1}(\infty)$ against $\lambda$, is depicted in Fig. 1, where $s_{1}=0.6$ and $s_{2}=0.4$. Observe that the maximum value of the variance, $\operatorname{Var}\left[p_{1}(\infty)\right]$ $=s_{1} s_{2}=0.24$, is attained when $\lambda=0$, and the minimum value of the variance, $\operatorname{Var}\left[p_{1}(\infty)\right]=0$, is achieved when $\lambda=1$.

Although the result derived is of an asymptotic sort, if the value of $\lambda$ is even as "small" as 0.9, the SLWE will be able to track the change, even if the environment switches its Bernoulli parameter after 50 steps. Observe too that we do not need to introduce or consider the use of a "sliding window".

\section{Weak Estimators of Multinomial Distributions}

In this section, we shall consider the problem of estimating the parameters of a multinomial distribution. The multinomial distribution is characterized by two parameters, namely, the number of trials, and a probability vector which determines the probability of a specific event (from a pre-specified set of events) occurring. In this regard, the number of observations is the number of trials. Thus, we are to estimate the latter probability vector associated with the set of possible outcomes or trials. Specifically, let $X$ be a multinomially distributed random variable, which takes on the values from the set $\{$ ' 1 ', . . ' $r$ ' $\}$. We assume that $X$ is governed by the distribution $S=\left[s_{1}, \ldots, s_{r}\right]^{T}$ as:

$X=' i$ ' with probability $s_{i}$, where $\sum_{i=1}^{r} s_{i}=1$.

Also, let $x(n)$ be a concrete realization of $X$ at time ' $n$ '. The intention of the exercise is to estimate $S$, i.e., $s_{i}$ for $i=1, \ldots, r$. We achieve this by maintaining a running estimate $P(n)=\left[p_{1}(n), \ldots, p_{r}(n)\right]^{T}$ of $S$, where $p_{i}(n)$ is the estimate of $s_{i}$ at time 


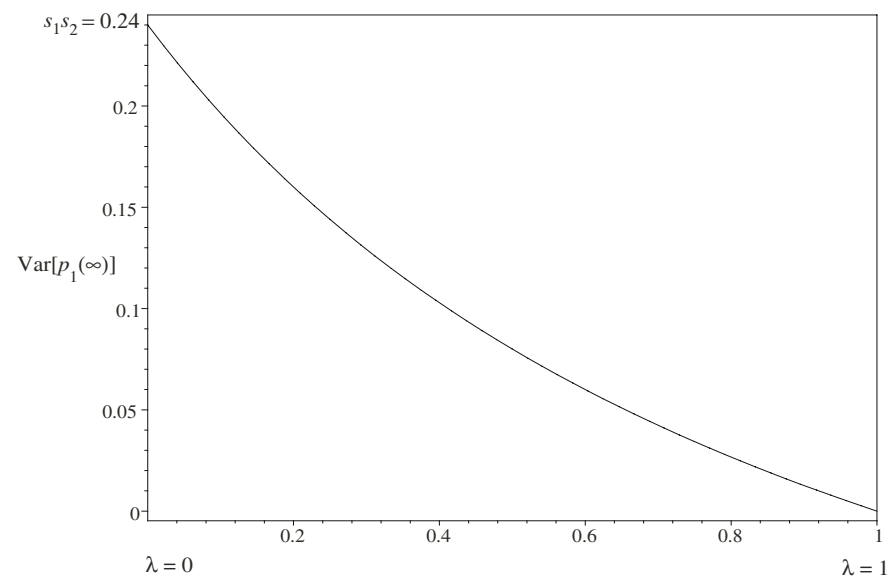

Fig. 1. Plot of the function $\operatorname{Var}\left[p_{1}(\infty)\right]$ for different values of $\lambda$ in the interval $[0,1]$, where $s_{1}=0.6$ and $s_{2}=0.4$.

' $n$ ', for $i=1, \ldots, r$. Then, the value of $p_{1}(n)$ is updated as per the following simple rule (the rules for other values of $p_{j}(n)$ are similar):

$$
\begin{array}{rlrl}
p_{1}(n+1) & \leftarrow p_{1}-(1-\lambda) \sum_{j \neq 1} p_{j} & & \text { when } x(n)=1 \\
& \leftarrow \lambda p_{1} & \text { when } x(n) \neq 1
\end{array}
$$

As in the binomial case, the vector $P(n)=\left[p_{1}(n), p_{2}(n), \ldots, p_{r}(n)\right]^{T}$ refers to the estimate of $S=\left[s_{1}, s_{2}, \ldots, s_{r}\right]^{T}$ at time ' $n$ ', and we will omit the reference to time ' $n$ ' in $P(n)$ whenever there is no confusion.

The results that we present now are as in the binomial case, i.e. the distribution of $\mathrm{E}[P(n+1)]$ follows $\mathrm{E}[P(n)]$ in a Markovian manner. This leads us to two results, namely to that of the limiting distribution of the chain, and that which concerns the rate of convergence of the chain. It turns out that both of these, in the very worst case, could only be dependent on the learning parameter $\lambda$. However, to our advantage, while the former is independent of the learning parameter, $\lambda$, the latter is only determined by it (and not a function of it). The complete proofs of Theorems 5, 6 and 7 are found in [12].

Theorem 5. Let $X$ be a multinomially distributed random variable governed by the distribution $S$, and $P(n)$ be the estimate of $S$ at time ' $n$ ' obtained by (3) and (4). Then, $E[P(\infty)]=S$.

We shall now state that the convergence of $P$ to $S$ occurs in a Markovian manner and derive the explicit form of the underlying Markovian matrix, say, $M$. Thus, we analyze the properties of $M$, and in particular, its eigenvalue properties.

Theorem 6. Let $X$ be a multinomially distributed random variable governed by the distribution $S$, and $P(n)$ be the estimate of $S$ at time ' $n$ ' obtained by (3) and (4). Then, 
the expected estimated probability vector follows a Markovian behavior in which every off-diagonal term of the underlying Markov matrix has the same multiplicative factor, $(1-\lambda)$. Furthermore, the final expected solution is independent of $\lambda$.

The convergence and eigenvalue properties of $\mathbf{M}$ follow.

Theorem 7. Let $X$ be a multinomially distributed random variable governed by the distribution $S$, and $P(n)$ be the estimate of $S$ at time ' $n$ ' obtained by (3) and (4). Then, all the non-unity eigenvalues of $\mathrm{M}$ are exactly $\lambda$, and thus the rate of converge of $P$ is fully determined by $\lambda$.

A small value of $\lambda$ leads to fast convergence and a large variance, and vice versa. Again, although the results we have derived are asymptotic, if $\lambda$ is even as "small" as 0.9 , after 50 iterations, the variation from the asymptotic value will be of the order of $10^{-50}$. In other words, after 50 steps, the SLWE will be able to track this change. Our experimental results demonstrate this fast convergence.

\section{Experimental Results}

To assess the efficiency of the SLWE introduced here, we have estimated the parameters for binomial and multinomial random variables using a value of $\lambda=0.99$. We have also estimated the parameters of these random variables by following the traditional MLE.

\subsection{Binomial Random Variables}

The estimation of the parameters for binomial random variables has been extensively tested for numerous distributions. In the interest of brevity, we merely cite one specific example. Also, to make the comparison meaningful, we have followed the MLE computation using the identical data stream. In each case, the estimation algorithms were presented with random occurrences of the variables for $n=4,000$ time instances. In the case of the SLWE, the true underlying value of $s_{1}$ was initially set to be 0.8 , and randomly modified after every 500 steps. The new values in the next periods were uniformly drawn from the interval $[0,1]$, being $0.42,0.92$, etc.

We report two kinds of results. In the first case, the results obtained are from a single run. The plot of this behavior is shown in Fig. 2. Observe that the MLE starts with an initial value of 0.5 and attains a value quite close to 0.8 in the first time period. As opposed to this, the SLWE starts close to the value 0.5, but quickly (in less than 100 iterations) converges to a value close to 0.8 . Thereafter, the MLE is not capable of tracking the "switching" of the Bernoulli parameter. Thus, at the end of the second period, during which the value of $s_{1}$ is 0.42 , the MLE attains a value of 0.638794 , which is significantly different from the true one. The SLWE, however, quickly learns that the parameter has changed, and its estimate, $p_{1}$, of $s_{1}$ is much closer to the true value. In the interest of playing on a level field, we have assumed that neither algorithm is aware of the switching nature of the environment. In other words, the MLE does not use a "sliding window" to estimate the running mean. The behavior of the MLE would probably be superior if this information were provided, but the question of how large 


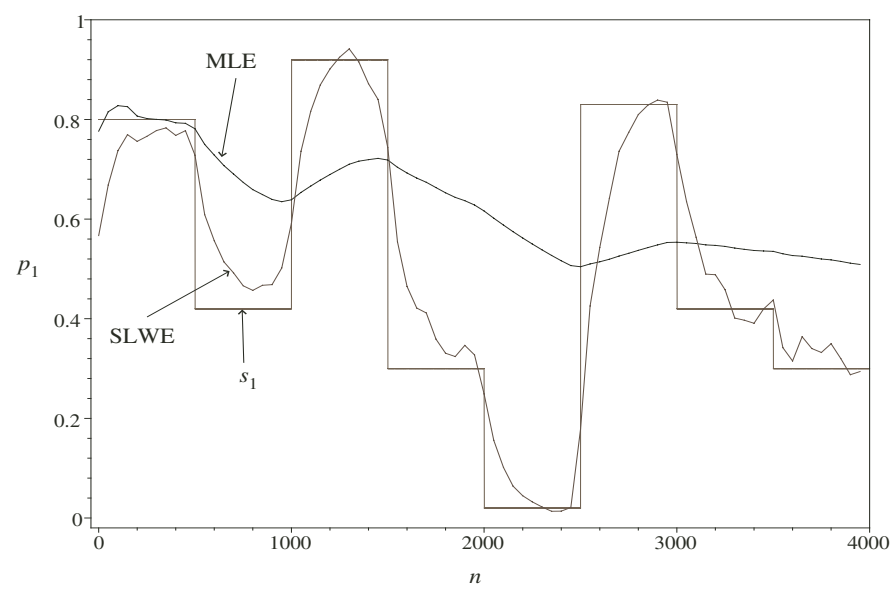

Fig. 2. Plot of the expected value of $p_{1}(n)$, at time ' $n$ ', which was estimated by using the SLWE and the MLE. The actual values of $s_{1}$, which change every 500 time steps, are also plotted.

this window should be, is a consideration that is not easily determined. This would be specially crucial in fast-changing environments, where the SLWE would not require any additional information.

In order to demonstrate the superiority of the SLWE over the MLE on an ensemble of experiments, we also report the respective ensemble averages. The same experiment was repeated 1,000 times, and the ensemble average at every time step was recorded. Clearly, the variations of the estimates would be much smoother. This behavior is shown in Fig. 3. Observe that the MLE follows $s_{1}$ exactly in the first window, but is thereafter severely handicapped in tracking the variations. In contrast, the SLWE quickly adjusts to the changes, as expected, in a geometric manner. In our opinion, the results are quite impressive. The plot of the "empirical" variance (shown in [12]) is identical.

\subsection{Multinomial Random Variables}

We have also performed simulations for multinomial random variables, where the parameters were estimated by following the SLWE and the MLE. We considered a multinomial random variable, $X$, which can take any of four different values, namely ' 1 ', '2', '3' or '4'. As in the binomial case, we ran the estimators for 4, 000 steps, and repeated this 1,000 times. We then took the ensemble average. For each experiment, we computed $\|P-S\|$, the Euclidean distance between $P$ and $S$. This was a measure of how good our estimate, $P$, was of $S$.

The plot of the latter distance obtained from our experiments is depicted in Fig. 4. While the distance of the SLWE quickly drops towards zero for every "switch" of $S$, the MLE is not able to capture these "switches" properly. Experimental results on real-life problems, involving adaptive data compression show a similar pattern [12]. 


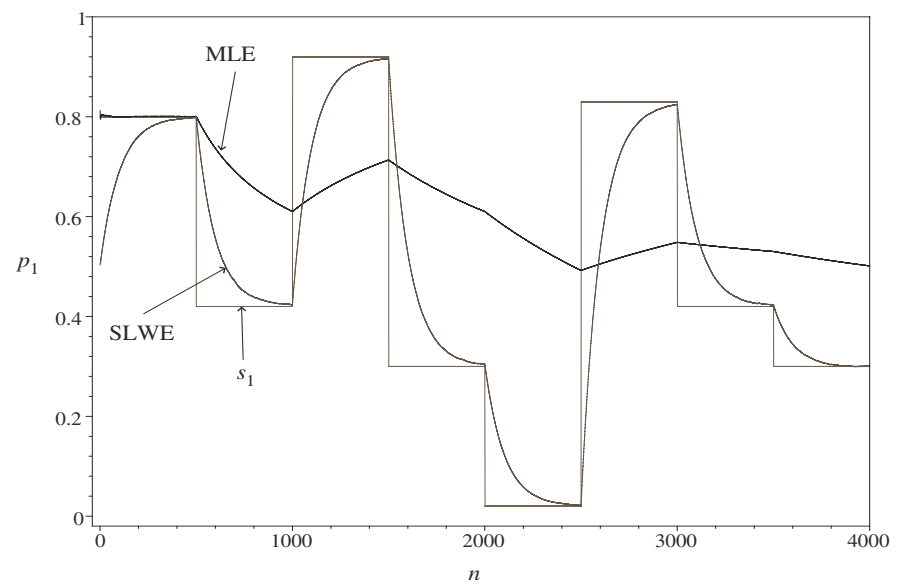

Fig. 3. Plot of the estimated probability of $s_{1}, p_{1}(n)$, at time ' $n$ ' by utilizing two approaches: the SLWE and the MLE. The actual values of $s_{1}$, which change every 500 time steps, are also plotted.

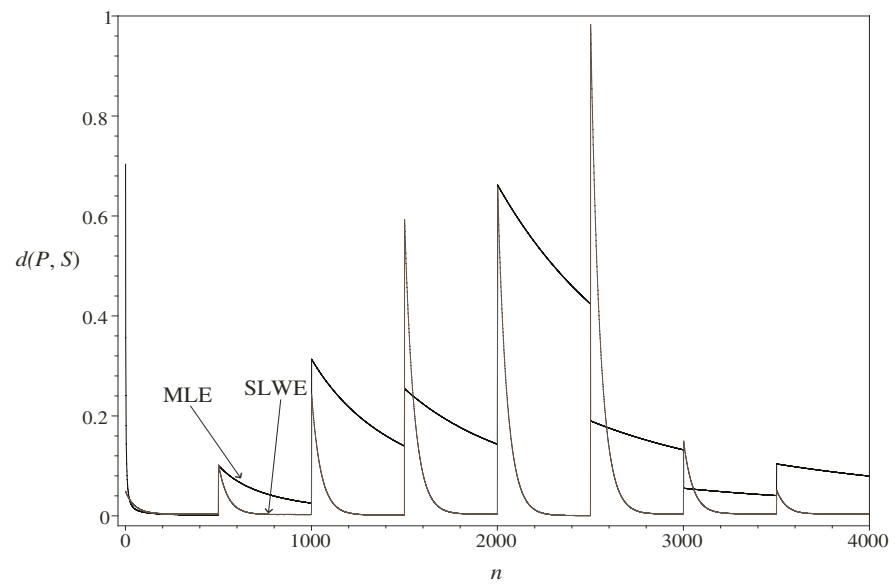

Fig. 4. Plot of the Euclidean norm of $P-S$ (or Euclidean distance between $P$ and $S$ ), where $P$ was estimated by using both the SLWE and the MLE. The values for the distances are computed for groups of 500 steps.

\section{Conclusions and Future Work}

In this paper, we have considered the problem of estimating the parameters of a distribution from its observations. Unlike traditional estimates that possess strong convergence properties, we have argued that there is a need for estimates that do not possess such strong convergence properties. Motivated by real-life applications, we formally presented an estimation method based on the principles of stochastic learning, which 
yields the binomial and multinomial estimates that converge weakly, i.e. in law. In the case of the binomial distribution, even though our new method includes a learning coefficient, $\lambda$, the mean of the final estimate is independent of $\lambda$, but the asymptotic variance decreases with $\lambda$, as does the convergence. Similar results are true for the multinomial case. Experimental results for both binomial and multinomial random variables, and for compressing data files drawn from non-stationary sources demonstrate the superiority of the SLWE over the MLE in these application domains.

\section{References}

1. G. Casella and R. Berger. Statistical Inference. Brooks/Cole Pub. Co., second edition, 2001.

2. R. Duda, P. Hart, and D. Stork. Pattern Classification. John Wiley and Sons, Inc., New York, NY, 2nd edition, 2000.

3. S. Lakshmivarahan. Learning Algorithms Theory and Applications. Springer-Verlag, New York, 1981.

4. S. Lakshmivarahan and M. A. L. Thathachar. Absolutely Expedient Algorithms for Stochastic Automata. IEEE Trans. on System, Man and Cybernetics, SMC-3:281-286, 1973.

5. J. K. Lanctôt and B. J. Oommen. Discretized Estimator Learning Automata. IEEE Trans. on Systems, Man and Cybernetics, 22(6):1473-1483, 1992.

6. K. Narendra and M. Thathachar. Learning Automata. An Introduction. Prentice Hall, 1989.

7. J. Norris. Markov Chains. Springer Verlag, 1999.

8. B.J. Oommen. Absorbing and Ergodic Discretized Two-Action Learning Automata. IEEE Trans. on System, Man and Cybernetics, SMC-16:282-296, 1986.

9. B.J. Oommen and M. Agache. Continuous and Discretized Pursuit Learning Schemes: Various Algorithms and Their Comparison. IEEE Trans. on Systems, Man and Cybernetics, SMC-31(B):277-287, June 2001.

10. B.J. Oommen and J. R. P. Christensen. Epsilon-Optimal Discretized Reward-Penalty Learning Automata. IEEE Trans. on System, Man and Cybernetics, SMC-18:451-458, 1988.

11. B.J. Oommen and J. K. Lanctôt. Discretized Pursuit Learning Automata. IEEE Trans. on System, Man and Cybernetics, 20(4):931-938, 1990.

12. B.J. Oommen and L. Rueda. Stochastic Learning-based Weak Estimation of Multinomial Random Variables and Its Applications to Non-stationary Environments. (Submitted for Publication).

13. G. I. Papadimitriou. A New Approach to the Design of Reinforcement Schemes for Learning Automata: Stochastic Estimator Learning Algorithms. IEEE Trans. on Knowledge and Data Engineering, 6:649-654, 1994.

14. K. Rajaraman and P. S. Sastry. Finite Time Analysis of the Pursuit Algorithm for Learning Automata. IEEE Trans. on Systems, Man and Cybernetics, 26(4):590-598, 1996.

15. S. Ross. Introduction to Probability Models. Academic Press, second edition, 2002.

16. M. A. L. Thathachar and P.S. Sastry. A Class of Rapidly Converging Algorithms for Learning Automata. IEEE Trans. on Systems, Man and Cybernetics, SMC-15:168-175, 1985.

17. A. Webb. Statistical Pattern Recognition. John Wiley \& Sons, N.York, second edition, 2002. 